\title{
Utilization of Digoxin among Hospitalized Older Patients with Heart Failure and Atrial Fibrillation in Thailand: Prevalence, Associated Factors, and Clinical Outcomes
}

\author{
Noppaket Singkham ${ }^{1,2}$, Yuttana Wongsalap ${ }^{1,2}$, Duangkamon Poolpun ${ }^{3}$, Sirichok Phetnoo ${ }^{1}$, Chuthalak Somkhon ${ }^{1}$ \\ ${ }^{1}$ Division of Pharmacy Practice, Department of Pharmaceutical Care, School of Pharmaceutical Sciences, University of Phayao, Phayao, Thailand \\ ${ }^{2}$ Unit of Excellence on Pharmacogenomic Pharmacokinetic and Pharmacotherapeutic Researches (UPPER), School of Pharmaceutical Sciences, University of \\ Phayao, Phayao, Thailand \\ ${ }^{3}$ Department of Pharmacy, Buddhachinaraj Hospital, Phitsanulok, Thailand
}

\section{Corresponding Author:}

Noppaket Singkham, $\mathrm{PhD}$

Division of Pharmacy Practice,

Department of Pharmaceutical Care,

School of Pharmaceutical Sciences,

University of Phayao, Tambon Maeka,

Muang District, Phayao Province

56000, Thailand

E-mail:noppaket.si@up.ac.th

ORCID:

https://orcid.org/0000-0003-2650-1121

Received: September 3, 2021

Revised: December 2, 2021

Accepted: December 7, 2021
Background: Digoxin is used to control heart rate in patients with heart failure (HF) and atrial fibrillation (AF). However, its use is often limited in older patients, as they are prone to digoxin toxicity. This study aimed to determine the prevalence of digoxin use, investigate the factors associated with digoxin use, and explore the association between digoxin use and clinical outcomes in older Thai patients with HF and AF. Methods: This cross-sectional study used data obtained from an electronic medical records database. We performed logistic regression analysis to determine the prevalence of digoxin use at index discharge and the factors associated with its use. The Cox proportional hazard model was used to determine the association of all-cause mortality and HF rehospitalization with digoxin use. Results: Of the 640 patients assessed, 107 (16.72\%) were prescribed digoxin before discharge. The factors negatively associated with digoxin use included high serum creatinine level (adjusted odds ratio $[A O R]=0.38 ; 95 \%$ confidence interval $[\mathrm{Cl}], 0.22-$ 0.65 ) and ischemic heart disease (IHD) ( $\mathrm{AOR}=0.52 ; 95 \% \mathrm{Cl}, 0.30-0.88)$. The factors positively associated with digoxin use were the use of diuretics (AOR=2.65; 95\% Cl, 1.60-4.38) and mineralocorticoid receptor antagonists (MRAs) ( $A O R=2.24 ; 95 \% \mathrm{Cl}, 1.18-4.27$ ). We observed no significant association between digoxin use and clinical outcomes (adjusted hazard ratio $=1.00 ; 95 \%$ $\mathrm{Cl}, 0.77-1.30)$. Conclusion: Digoxin use was prevalent among older patients with HF and AF. Patients with high serum creatinine or IHD were less likely to be prescribed digoxin, whereas those using diuretics or MRAs were more likely to be prescribed digoxin. Although digoxin use was not uncommon among older patients, it was prescribed with caution among Thai patients hospitalized with $\mathrm{HF}$ and $\mathrm{AF}$.

Key Words: Frail older adults, Prevalence, Drug utilization, Digoxin, Heart failure, Atrial fibrillation

\section{INTRODUCTION}

Atrial fibrillation (AF) typically coexists with heart failure (HF), especially in older adults. The coexistence of both conditions at the time of discharge from the hospital is frequently reported among hospitalized older adults. ${ }^{1,2)}$ The prevalence of AF among patients with HF increases with age. ${ }^{1,3)}$ A report on older Thai patients (mean age, $64 \pm 14$ years) hospitalized for acute HF revealed that $24.1 \%$ had a history of $\mathrm{AF}^{4)}$ Additionally, combined $\mathrm{HF}$ and AF occurred in $20.8 \%$ of patients aged $\geq 65$ years. ${ }^{5)}$ The coexistence of both conditions in older adults was not only associated with increased adverse outcomes such as mortality, hospitalization, 
and healthcare costs ${ }^{4,5)}$ but also necessitated complicated pharmacological therapies. ${ }^{1)}$

Digoxin is a therapeutic agent used for the treatment of HF and AF. Its use is dependent on the type of HF. HF is classified by ejection fraction (EF) as HF with reduced $\mathrm{EF}$ ( $\mathrm{HFrEF})(\mathrm{EF}<40 \%)$, HF with mid-range EF (HFmrEF) (EF 41\%-49\%), and HF with preserved $\mathrm{EF}(\mathrm{HF} p \mathrm{EF})(\mathrm{EF} \geq 50 \%)$. In the treatment of $\mathrm{HF}$ alone, digoxin is recommended as an add-on therapy in symptomatic patients with HFrEF to reduce HF-related hospitalization but is not recommended for use in patients with HFmrEF and HFpEF due to a lack of clinical benefit. ${ }^{6-8)}$ However, digoxin still plays an important role in AF therapy in patients with HF. In the treatment of $\mathrm{AF}$ in these patients, the three common therapeutic agents recommended for heart rate control are beta-blockers (BBs), non-dihydropyridine calcium channel blockers (non-DHP CCBs), and digoxin. ${ }^{9-11)}$ While any of these three options can be used in patients with $\mathrm{HF} m r \mathrm{EF}$ and $\mathrm{HF} p \mathrm{EF}$, non-DHP CCBs are contraindicated in patients with $\mathrm{HFrEF}{ }^{9,11)}$ Although BBs and non-DHP CCBs are considered first-line therapy, their synergistic negative inotropic effect on the heart is a significant clinical concern for older adults, who are vulnerable to this effect. Avoidance of non-DHP CCBs is also recommended for patients with severe $\mathrm{HF}^{12)}$ Thus, digoxin has become an alternative treatment choice for older patients with both $\mathrm{HF}$ and $\mathrm{AF}$, resulting in its broad prescription in clinical practice.

The prevalence of digoxin use in previous studies varied depending on the study population. Digoxin was more likely to be prescribed to patients with $\mathrm{HF}$ and $\mathrm{AF}$ than to patients with $\mathrm{HF}$ alone. For example, prevalence rates of $20.84 \%{ }^{13)}$ and $11.0 \%{ }^{14)}$ were reported in patients with $\mathrm{HF}$ with and without $\mathrm{AF}$, respectively. This is because digoxin is used as an add-on therapy in HFrEF but is used as mainstream therapy in AF. Nevertheless, digoxin use is not considered to be highly prevalent in patients with both conditions. Advanced age and renal function are important factors that limit the use of digoxin in clinical practice because they are associated with an increased risk of digoxin toxicity that is common among older patients and patients with impaired renal function.

Among clinical outcomes, a randomized trial demonstrated the relationship between digoxin treatment and decreased risk of rehospitalization in HFrEF patients without concurrent $\mathrm{AF}^{15}$ and that the relationship was not age-dependent. ${ }^{16)}$ However, the clinical benefits of digoxin for older adults with both HF and AF remain controversial. Both post-hoc analyses of randomized controlled trials (RCTs) ${ }^{17-19)}$ and well-designed observational studies $^{13,20-25)}$ have reported conflicting evidence on the risk-benefit profile of digoxin. Some studies observed an association of digoxin and increased risk of mortality in AF patients with or without $\mathrm{HF}^{17-20)}$ Conversely, several other studies did not report such find- ings. ${ }^{13,21-25)}$ Recently, studies in Asians have evaluated the safety and benefits of digoxin use in older patients. ${ }^{26,27)}$ A study in Taiwan showed that digoxin use was related to increased ischemic stroke and mortality in AF patients without HF but not in those with $\mathrm{HF}^{26)}$ Another study in China found that digoxin use was related to an increased likelihood of mortality and rehospitalization in HFrEF patients with or without $\mathrm{AF}^{27)}$ These results varied according to differences in the prevalence of digoxin use and outcomes in each clinical setting.

In Thailand, the national HF guidelines suggest the use of digoxin as a therapeutic agent for heart rate control in patients with HF and coexisting $\mathrm{AF}^{9)}$ However, the prescribing rate, contributing factors, and clinical outcomes of digoxin use in older adults with both conditions have not been reported. Thus, the primary objective of the present study was to determine the prevalence and identify factors associated with digoxin use. The secondary objective was to explore the association between digoxin treatment and clinical outcomes.

\section{MATERIALS AND METHODS}

\section{Study Design and Settings}

This cross-sectional study included data on patients diagnosed with both HF and AF, which were obtained from the electronic medical records (EMR) database of a tertiary care teaching urban hospital (a 1,100-bed public hospital) in Phitsanulok Province. The study hospital is an academic referral center for the lower northern region of Thailand. The EMR database included patient demographics, clinical characteristics, and medication information. At the time of the study, well-trained hospital staff entered patient information into the EMR database.

The research protocol was approved by the Research Ethics Committee of Buddhachinaraj Hospital (IRB No. 067/63, Approval data: March 26, 2020) before retrieval of patient data.

\section{Subjects}

The analysis included patients aged $\geq 60$ years (based on a definition of "older adult" of 60 years) ${ }^{28)}$ and diagnosed with $\mathrm{HF}$ and AF who were hospitalized for HF for the first time between January 2014 and December 2018 (defined as the patient's index hospitalization). Patients were identified as having $\mathrm{HF}$ and $\mathrm{AF}$ using the following International Statistical Classification of Disease and Related Health Problems 10th Revision (ICD-10) codes: I50.0, I50.1, or I50.9 (for HF) and I48.0 (for AF). Patients with serum creatinine concentrations $>3 \mathrm{mg} / \mathrm{dL}$ or serum potassium concentrations $<3.2$ or $>5.5 \mathrm{mEq} / \mathrm{L}$ were excluded from the analysis.

Among the eligible patients discharged alive, those who were 
prescribed oral digoxin at index predischarge were assigned to the exposed group (i.e., patients treated with digoxin). The remaining patients were assigned to the unexposed group (i.e., patients not treated with digoxin). The patients in the unexposed group were examined for digoxin prescription post-discharge to ensure that none were exposed to digoxin during the follow-up period. Both patient groups were followed up until December 10, 2020, for clinical outcome measurement.

\section{Data Collection}

All study factors and outcomes were obtained from the EMR database. The study factors were categorized as patient demographics, including sex and age (calculated on the index date of admission); clinical characteristics, including echocardiogram results, last laboratory findings, length of stay (LOS); cardiovascular (CV) comorbidities and comorbidity score calculated using the Charlson Comorbidity Index (CCI); ${ }^{29,30)}$ and discharge medication lists. Data on disease severity (e.g., New York Heart Association [NYHA] classification or brain natriuretic peptide [BNP] level) were not routinely recorded in the EMR database. As one study suggested that LOS, which might indicate HF severity or complex conditions, was a positive predictor of rehospitalization, ${ }^{31)}$ we used this factor to determine HF severity in this study. All medications used for the treatment of AF and HF were identified using the drug codes of the study hospital.

\section{Definitions of Clinical Outcomes}

The primary outcome was a composite outcome of all-cause mortality and HF rehospitalization occurring after the index discharge. The primary discharge diagnosis, which was defined based on ICD-10 codes related to HF diagnoses (I50.0, I50.1, or I50.9), was used to identify HF rehospitalization. The cause of death, which was recorded in the database, was used to identify in-hospital deaths from any cause. The secondary outcomes were all-cause mortality and HF rehospitalization. Digoxin toxicity was identified according to ICD-10 code T46.0.

\section{Statistical Analysis}

Continuous variables with normal distribution were presented as mean \pm standard deviation, and independent sample t-tests were used for comparisons between patient groups. Continuous variables with non-normal distribution were presented as median and interquartile range (IQR), and Mann-Whitney $U$ tests were used for between-group comparisons. Categorical variables were presented as frequencies and percentages, and either the chi-square test or Fisher exact test was used for between-group comparisons, as appropriate.
The prevalence of digoxin use was calculated by dividing the number of patients who were prescribed digoxin by the total number of patients and presented as a percentage (\%). To determine the factors affecting digoxin prescription, we performed univariable and multivariable binary logistic regression analyses and calculated crude odds ratios (ORs), adjusted ORs, and 95\% confidence intervals (CIs). In univariate analysis, we selected factors with $\mathrm{p}<0.1$ for multicollinearity tests. In the multicollinearity tests, in which independent variables in a binary logistic regression model are highly correlated, we excluded factors with a variance inflation factor $(\mathrm{VIF})>10 .^{32}$ In the multivariate analysis, we applied the backward elimination method, in which the least significant variable was discarded at each step until all remaining variables in the model reached a significance level of $\leq 0.05$.

We also assessed clinical outcomes, including all-cause mortality and rehospitalization due to $\mathrm{HF}$, and calculated hazard ratios (HRs) using the Cox proportional hazards model between patients treated and not treated with digoxin. The cohorts were adjusted using the most frequently reported factors associated with all-cause mortality and rehospitalization due to $\mathrm{HF}$, including sex, age, comorbidity score, and LOS. All analyses were conducted using Stata release 14.0 (Stata Corporation, College Station, TX, USA). Statistical significance was set at $\mathrm{p}<0.05$. All $\mathrm{p}$-values were two-tailed.

\section{RESULTS}

\section{Patient Characteristics}

From the EMR database, we included a total of 640 older adults with $\mathrm{HF}$ and $\mathrm{AF}$ (Fig. 1). Characteristics of the total patient group,

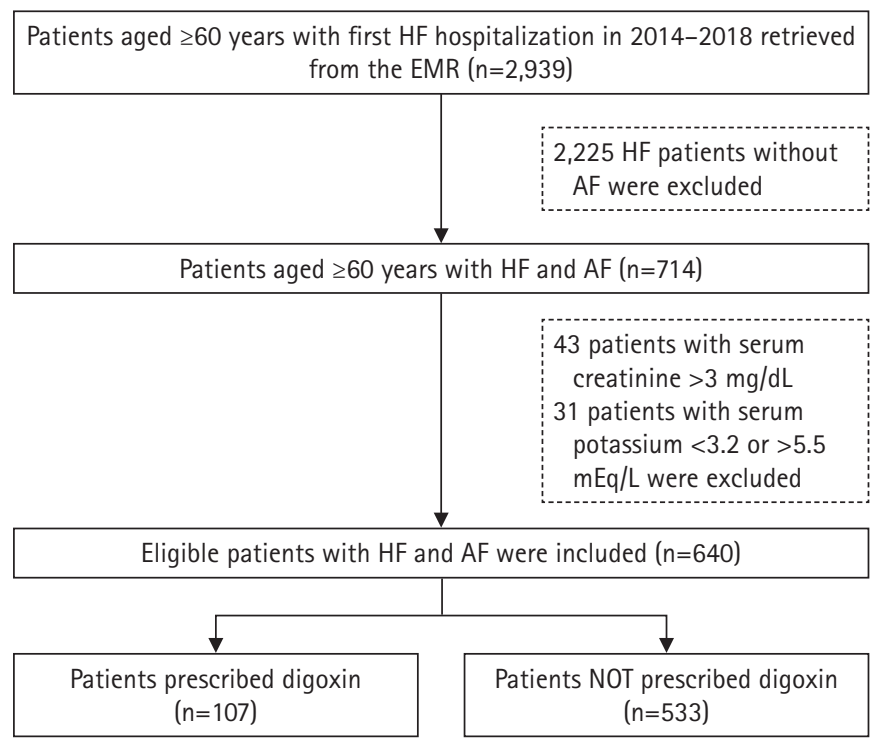

Fig. 1. Flowchart showing the process of data retrieval. HF, heart failure; EMR, electronic medical records; AF, atrial fibrillation. 
patients treated with digoxin, and those not treated with digoxin are shown in Table 1. Overall, the average age of the patients was $74.42 \pm 8.23$ years and they were mostly female (55.62\%). Among patients with EF (46.72\%), almost three-fourths had an EF $\geq 40 \%$. The mean serum potassium and serum creatinine concentrations were $3.9 \pm 0.42 \mathrm{mmol} / \mathrm{L}$ and $1.25 \pm 0.50 \mathrm{mmol} / \mathrm{L}$, respec- tively.

The most frequently reported cardiovascular comorbidity was hypertension, followed by dyslipidemia and ischemic heart disease (IHD). Almost all patients (93.44\%) were treated with oral anticoagulants (OACs), and BBs were the primary agents used to control heart rate. Among HF medications, 44.84\%, 36.57\%, and

Table 1. Characteristics of all patients with heart failure and atrial fibrillation

\begin{tabular}{|c|c|c|c|c|}
\hline Characteristic & Total patients $(\mathrm{n}=640)$ & Patients treated with digoxin $(\mathrm{n}=107)$ & Patients not treated with digoxin $(\mathrm{n}=533)$ & p-value \\
\hline \multicolumn{5}{|l|}{ Demographics } \\
\hline Sex, male & $284(44.38)$ & $42(39.25)$ & $242(45.40)$ & 0.243 \\
\hline Age (y) & $74.42 \pm 8.23$ & $72.94 \pm 7.75$ & $74.71 \pm 8.33$ & 0.044 \\
\hline$\geq 80$ & $193(30.16)$ & $24(22.43)$ & $169(31.71)$ & 0.058 \\
\hline \multicolumn{5}{|l|}{ Clinical characteristics } \\
\hline $\mathrm{EF}^{\mathrm{a})}(\%)$ & $53.97 \pm 18.84$ & $51.44 \pm 18.15$ & $54.54 \pm 18.98$ & 0.271 \\
\hline HF with EF & & & & 0.666 \\
\hline $\operatorname{HFrEF}(\mathrm{EF}<40 \%)$ & $84(28.09)$ & $17(30.91)$ & $67(27.46)$ & \\
\hline HFmrEF (EF 40\%-49\%) & $31(10.37)$ & $4(7.27)$ & $27(11.07)$ & \\
\hline $\operatorname{HFpEF}(\mathrm{EF} \geq 50 \%)$ & $184(61.54)$ & $34(61.82)$ & $150(61.48)$ & \\
\hline Serum sodium $(\mathrm{mmol} / \mathrm{L})$ & $138.28 \pm 4.58$ & $137.83 \pm 4.79$ & $138.37 \pm 4.53$ & 0.266 \\
\hline Serum potassium $(\mathrm{mmol} / \mathrm{L})$ & $3.90 \pm 0.42$ & $3.89 \pm 0.40$ & $3.90 \pm 0.42$ & 0.920 \\
\hline Serum creatinine $(\mathrm{mg} / \mathrm{dL})$ & $1.25 \pm 0.50$ & $1.08 \pm 0.36$ & $1.28 \pm 0.52$ & $<0.001$ \\
\hline $\mathrm{eGFR}(\mathrm{mL} / \mathrm{min})$ & $56.19 \pm 22.54$ & $62.76 \pm 19.67$ & $54.87 \pm 22.86$ & 0.001 \\
\hline LOS (day) & $6(3-10)$ & $5(4-9)$ & $6(3-10)$ & 0.532 \\
\hline$>5$ & $331(51.72)$ & $53(49.53)$ & $278(52.16)$ & 0.620 \\
\hline \multicolumn{5}{|l|}{ Cardiovascular comorbidities } \\
\hline Hypertension & $278(43.44)$ & $41(38.32)$ & $237(44.47)$ & 0.242 \\
\hline Dyslipidemia & $198(30.94)$ & $27(25.23)$ & $171(32.08)$ & 0.163 \\
\hline Ischemic heart disease & $189(29.53)$ & $21(19.63)$ & $168(31.52)$ & 0.015 \\
\hline Diabetes mellitus & $157(24.53)$ & $24(22.43)$ & $133(24.95)$ & 0.580 \\
\hline Chronic kidney disease & $50(7.81)$ & $5(4.67)$ & $45(8.44)$ & 0.192 \\
\hline Stroke & $12(1.88)$ & $1(0.93)$ & $11(2.06)$ & 0.444 \\
\hline Comorbidity score $\geq 2$ & $350(54.69)$ & $53(49.53)$ & $297(55.72)$ & 0.015 \\
\hline Comorbidity score & $2(1-2)$ & $1(1-2)$ & $2(1-2)$ & 0.241 \\
\hline \multicolumn{5}{|l|}{ HF medications } \\
\hline OACs & $598(93.44)$ & $107(100.00)$ & $491(92.12)$ & 0.003 \\
\hline Diuretics & $391(61.09)$ & $83(77.57)$ & $308(57.79)$ & $<0.001$ \\
\hline BBs & $287(44.84)$ & $43(40.19)$ & $244(45.78)$ & 0.289 \\
\hline ACEIs & $172(26.88)$ & $37(34.58)$ & $135(25.33)$ & 0.050 \\
\hline ARBs & $62(9.69)$ & $14(13.08)$ & $48(9.01)$ & 0.196 \\
\hline MRAs & $51(7.97)$ & $18(16.82)$ & $33(6.19)$ & $<0.001$ \\
\hline Nitrates & $50(7.81)$ & $7(6.54)$ & $43(8.07)$ & 0.592 \\
\hline DHP CCBs & $41(6.41)$ & $4(3.74)$ & $37(6.94)$ & 0.224 \\
\hline Hydralazine & $30(4.69)$ & $2(1.87)$ & $28(5.25)$ & 0.149 \\
\hline Non-DHP CCBs & $18(2.81)$ & $5(4.67)$ & $13(2.44)$ & 0.210 \\
\hline
\end{tabular}

Values are presented as number (\%) or mean \pm standard deviation or median (interquartile range).

HFrEF, heart failure with reduced ejection fraction; HFmrEF, heart failure with mid-range ejection fraction; HFpEF, heart failure with preserved ejection fraction; eGFR, estimated glomerular filtration rate; LOS, length of stay; OACs, oral anticoagulants; BBs, beta-blockers; ACEIs, angiotensin-converting enzyme inhibitors; ARBs, angiotensin II receptor blockers; CCBs, calcium channel blockers; MRAs, mineralocorticoid receptor antagonists.

${ }^{\text {a) }}$ Data were available for 55 and 244 patients with and without digoxin, respectively. 
7.97\% of the patients were receiving BBs, angiotensin-converting enzyme inhibitors (ACEIs) or angiotensin II receptor blockers (ARBs), and mineralocorticoid receptor antagonists (MRAs), respectively.

\section{Prevalence and Associated Factors of Digoxin Use}

Our review of the index discharge medication lists of all study patients showed that 107 patients $(16.72 \%)$ were prescribed oral digoxin at the index hospital discharge. The prevalence rate of digoxin use in patients with $\mathrm{EF}<40 \%$, with $\mathrm{EF} \geq 40 \%$, and with no $\mathrm{EF}$ were $20.24 \%, 17.67 \%$, and $15.25 \%$, respectively. Among patients treated with digoxin, most $(90.65 \%)$ received oral digoxin at a daily dose of $0.125 \mathrm{mg}$, and only a few received a daily dose of 0.0625 $\mathrm{mg}$ and $0.250 \mathrm{mg}$ (4.67\% each).

The results of univariate and multivariate analyses are shown in Table 2. In the univariate analysis, the factors negatively associated with digoxin use included older age (crude OR $=0.97$; 95\% CI, 0.95-0.99), higher serum creatinine (crude OR $=0.37$; $95 \% \mathrm{CI}$, $0.22-0.62$ ), IHD (crude OR=0.53; 95\% CI 0.32-0.88), and higher CCI (crude OR=0.74; 95\% CI, 0.59-0.95). The factors positively associated with digoxin use were higher estimated glomerular filtration rate (eGFR) (crude $\mathrm{OR}=1.02 ; 95 \% \mathrm{CI}, 1.01-1.03$ ), use of diuretics (crude OR $=2.53$; 95\% CI, 1.55-4.11), and use of MRAs (crude OR=3.06; 95\% CI, 1.65-5.68).

In the multivariate analysis, the factors negatively associated with digoxin use included higher serum creatinine (adjusted $\mathrm{OR}=0.38$; 95\% CI, 0.22-0.65) and IHD (adjusted $\mathrm{OR}=0.52$; $95 \%$ CI, 0.30-0.88). The two medication classes positively associated with digoxin use were diuretics and MRAs (adjusted $\mathrm{OR}=2.65 ; 95 \% \mathrm{CI}, 1.60-4.38$ and adjusted $\mathrm{OR}=2.24 ; 95 \% \mathrm{CI}$, $1.18-4.27$, respectively).

\section{Relationships between Digoxin Treatment and Clinical Outcomes}

The results of the analysis of the associations between digoxin treatment and clinical outcomes are shown in Table 3. The study outcomes occurred in 413 patients $(64.53 \%)$ during a median follow-up period of 4.7 (IQR 3.3-5.8) years. In an unadjusted analysis, we observed no statistically significant decrease in the risk of the primary outcome among patients treated with digoxin compared to the risk in those who did not receive digoxin $(\mathrm{HR}=0.91$; 95\% CI, 0.70-1.18). Patients treated with digoxin had a longer mean survival time ( $850 \pm 69$ vs. $789 \pm 30$ days; $p=0.414)$. In addition, we observed no statistically significant association after adjusting for sex, age, comorbidity score, and LOS (adjusted $\mathrm{HR}=1.00$; 95\% CI, 0.77-1.30) (Fig. 2).

Among the secondary outcomes, all-cause mortality and rehospitalization due to HF occurred in 372 (58.13\%) and 139 (21.72\%) patients, respectively. In the univariate analysis, we observed no statistically significant decreases in the risks of all-cause mortality (crude $\mathrm{HR}=0.91 ; 95 \% \mathrm{CI}, 0.70-1.83$ ) and rehospitalization due to $\mathrm{HF}$ (crude $\mathrm{HR}=0.99 ; 95 \% \mathrm{CI}, 0.64-1.53$ ) in patients treated with digoxin compared to those in patients who did not receive this treatment. After controlling for pre-specified covariates, the association remained the same for all-cause mortality (adjusted $\mathrm{HR}=1.02 ; 95 \% \mathrm{CI}, 0.77-1.35)$ and rehospitalization due to $\mathrm{HF}$ (crude $\mathrm{HR}=0.96 ; 95 \% \mathrm{CI}, 0.62-1.50$ ).

A subgroup analysis performed according to EF categories showed that treatment with digoxin was not significantly associated with the primary outcome in patients with $\mathrm{EF}<40 \%$ and in patients with $\mathrm{EF} \geq 40 \%$ (adjusted $\mathrm{HR}=0.87$; 95\% CI, 0.43-1.76; $\mathrm{p}=0.692$ and adjusted $\mathrm{HR}=1.10 ; 95 \% \mathrm{CI}, 0.71-1.71 ; \mathrm{p}=0.669$, respectively). Similarly, we observed no statistically significant association between treatment with digoxin and all-cause mortality

Table 2. Results of univariable and multivariable analyses for the identification of study factors associated with digoxin treatment

\begin{tabular}{|c|c|c|c|c|}
\hline Study factors ${ }^{a)}$ & Crude OR $(95 \%$ CI $)$ & p-value & Adjusted OR (95\% CI) & $\mathrm{p}$-value \\
\hline Age (y) & $0.97(0.95-0.99)$ & 0.044 & & \\
\hline Age, $\geq 80 y$ & $0.62(0.38-1.02)$ & 0.058 & & \\
\hline Serum creatinine $(\mathrm{mg} / \mathrm{dL})$ & $0.37(0.22-0.62)$ & $<0.001$ & $0.38(0.22-0.65)$ & $<0.001^{\mathrm{b})}$ \\
\hline eGFR (mL/min) & $1.02(1.01-1.03)$ & 0.001 & & \\
\hline Ischemic heart disease & $0.53(0.32-0.88)$ & 0.015 & $0.52(0.30-0.88)$ & $0.015^{\mathrm{b})}$ \\
\hline Comorbidity score & $0.74(0.59-0.95)$ & 0.015 & & \\
\hline ACEIs & $1.56(0.99-2.42)$ & 0.050 & & \\
\hline MRAs & $3.06(1.65-5.68)$ & $<0.001$ & $2.24(1.18-4.27)$ & $0.014^{\mathrm{b})}$ \\
\hline
\end{tabular}

OR, odds ratio; CI, confidence interval; eGFR, estimated glomerular filtration rate; ACEIs, angiotensin-converting enzyme inhibitors; MRAs, mineralocorticoid receptor antagonists.

${ }^{\text {a) }}$ Factors with p-value $<0.1$ were included in the multivariable model.

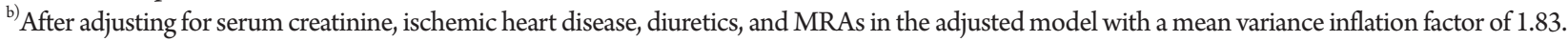


Table 3. Results of the analysis of associations between digoxin treatment and clinical outcomes

\begin{tabular}{|c|c|c|c|c|}
\hline Study outcomes & Crude HR $(95 \%$ CI $)$ & p-value & Adjusted $\mathrm{HR}^{\mathrm{a})}(95 \% \mathrm{CI})$ & p-value \\
\hline \multicolumn{5}{|c|}{ All-cause mortality and HF rehospitalization } \\
\hline All patients & $0.91(0.70-1.18)$ & 0.484 & $1.00(0.77-1.30)$ & 0.997 \\
\hline $\mathrm{EF}^{\mathrm{b})}<40 \%$ & $0.76(0.38-1.50)$ & 0.422 & $0.87(0.43-1.76)$ & 0.692 \\
\hline $\mathrm{EF} \geq 40 \%$ & $1.15(0.75-1.76)$ & 0.521 & $1.10(0.71-1.71)$ & 0.669 \\
\hline \multicolumn{5}{|c|}{ All-cause mortality } \\
\hline All patients & $0.91(0.70-1.83)$ & 0.507 & $1.02(0.77-1.35)$ & 0.874 \\
\hline $\mathrm{EF} \geq 40 \%$ & $1.06(0.67-1.68)$ & 0.813 & $1.09(0.68-1.74)$ & 0.730 \\
\hline \multicolumn{5}{|c|}{ HF rehospitalization } \\
\hline All patients & $0.99(0.64-1.53)$ & 0.963 & $0.96(0.62-1.50)$ & 0.872 \\
\hline $\mathrm{EF}<40 \%$ & $0.95(0.35-2.57)$ & 0.924 & $0.99(0.35-2.74)$ & 0.979 \\
\hline $\mathrm{EF} \geq 40 \%$ & $1.04(0.48-2.23)$ & 0.923 & $0.82(0.38-1.80)$ & 0.628 \\
\hline
\end{tabular}

HR, hazard ratio; CI, confidence interval; HF, heart failure; EF, ejection fraction.

${ }^{a}$ When controlling for sex, age, comorbidity score, and length of stay.

${ }^{b)} \mathrm{EF}$ data were available for 84 and 215 patients with $\mathrm{EF}<40 \%$ and $\geq 40 \%$, respectively.

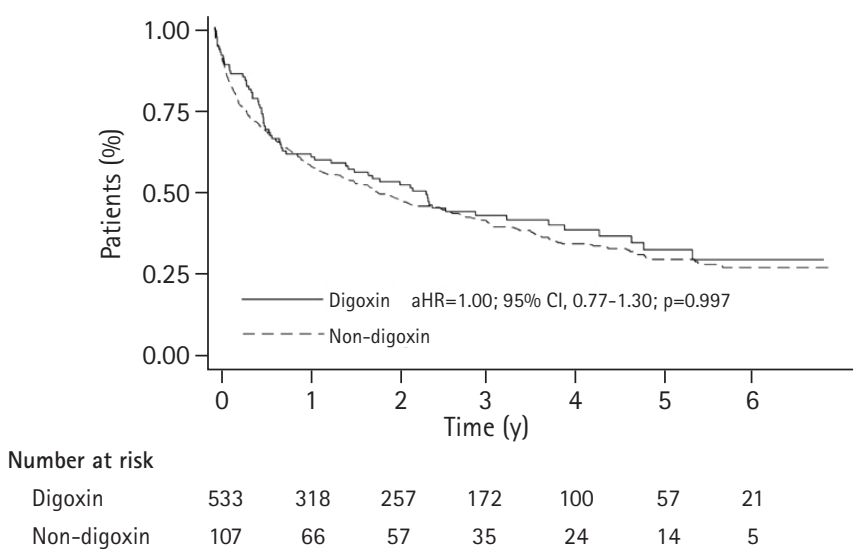

Fig. 2. Kaplan-Meier survival plot of all-cause mortality and heart failure rehospitalization according to digoxin prescription. aHR, adjusted hazard ratio; $\mathrm{CI}$, confidence interval.

and $\mathrm{HF}$ rehospitalization in patients with $\mathrm{EF}<40 \%$ and $\mathrm{EF} \geq 40 \%$ (Table 3).

\section{DISCUSSION}

The safety of digoxin treatment for heart rate control in older patients with $\mathrm{HF}$ and AF has been questioned due to its adverse clinical effects, including mortality, hospitalization, and digoxin toxicity. Given the safety concerns in this population, direct evidence on digoxin treatment among older Thai patients with both conditions is required to understand digoxin use in real-world clinical practice. Our results indicated that digoxin use was relatively prevalent. Both positively and negatively associated factors contributed to the use of digoxin. However, we observed no sta- tistically significant association between adverse clinical outcomes and digoxin use.

Despite advances in procedure-based AF therapies, pharmacological therapy still plays a major role in controlling the heart rate in AF treatment. The results of the present study revealed that BBs and digoxin were the primary agents for heart rate control in the study cohort, with $44.84 \%, 16.72 \%$, and $6.72 \%$ of patients receiving BBs, digoxin, and a combination of both agents, respectively, whereas only $2.81 \%$ of patients received non-DHP CCBs (verapamil and diltiazem). According to the AF status in HF guidelines, either $\mathrm{BBs}$, digoxin, or a combination can be used in all $\mathrm{HF}$ types ( $\mathrm{EF}<40 \%$ and $\geq 40 \%$ ), while non-DHP CCBs should be avoided in patients with $\mathrm{EF}<40 \%$. ${ }^{9)}$ Less than half of the patients (46.72\%) in this study had known EF, with $28.09 \%$ and $71.91 \%$ having EF $<40 \%$ and $\mathrm{EF} \geq 40 \%$, respectively. These data suggested that BBs and digoxin were safer than non-DHP CCBs for older patients with known and unknown EF. Additionally, the combination of BBs and non-DHP CCBs is inappropriate for older patients with $\mathrm{EF} \geq 40 \%$ because of their negative inotropic effect, which is high among these patients. ${ }^{12)}$ This is why digoxin is the second choice of therapeutic agent for heart rate control in older patients with $\mathrm{HF}$ and $\mathrm{AF}$.

The prevalence of digoxin use in our study was slightly lower than that reported previously (16.72\% vs. $20.84 \%) .{ }^{13)}$ Although we excluded patients with a contraindication to digoxin use (serum creatinine concentration $>3 \mathrm{mg} / \mathrm{dL}$ or serum potassium concentration $<3.2$ or $>5.5 \mathrm{mEq} / \mathrm{L}$ ), we identified other factors contributing to digoxin. Patients with higher serum creatinine concentration or IHD were less likely to be treated with digoxin, whereas patients receiving diuretics or MRAs were more likely to be treated 
with digoxin. Digoxin might not be considered for patients with increasing serum creatinine levels, which indicates worse renal function and a susceptibility to digoxin toxicity. ${ }^{33)}$ IHD may deteriorate due to the positive inotropic effect of digoxin. In addition, a recent study indicated that digoxin therapy was associated with an increased risk of all-cause and cardiovascular death in patients with ischemic heart failure. ${ }^{34)}$ Two medication classes were positively associated with digoxin use, including diuretics and MRAs. Current $\mathrm{HF}$ guidelines suggest digoxin as an adjunct to medical therapy for HFrEF; thus, digoxin may have been additionally prescribed in $\mathrm{HFrEF}$ patients receiving diuretics or MRAs, indicating persistent symptoms. ${ }^{9-11)}$ In addition to the previously mentioned factors, the low prevalence of digoxin use may be related to the clinicians' perception of increased mortality risk associated with digoxin use, as reported in previous studies. ${ }^{17-20)}$

Among adverse clinical impacts of digoxin use, we observed no occurrence of digoxin toxicity in patients treated with digoxin. This is because most of the patients in our study were administered a daily dose of $0.125 \mathrm{mg}$, which is the recommended oral digoxin dose for older patients with $\mathrm{HF}^{12)}$ We observed no significant relationship between digoxin treatment and all-cause mortality and HF rehospitalization in either unadjusted or adjusted analyses. Our study findings were consistent with those of previous studies that reported statistically insignificant results. ${ }^{13,21-25)}$ A retrospective propensity-matched analysis of a randomized trial found no evidence of increased mortality or hospitalization in patients with paroxysmal and persistent $\mathrm{AF}$ who received digoxin as baseline initial therapy. ${ }^{21)}$ In another observational propensity-matched study conducted by Singh et al., ${ }^{13)}$ the authors reported that digoxin initiation was not positively related to mortality and $\mathrm{HF}$ readmission in hospitalized older patients with $\mathrm{HF}$ ( $\mathrm{HFrEF}$ and $\mathrm{HF} p \mathrm{EF}$ ) and AF. Likewise, a previous study using data obtained from real clinical settings found no effect of digoxin use on mortality in AF patients with $\mathrm{HF}^{23)}$ Although these observational studies applied several methods for controlling potential confounding factors, including propensity matching, multivariable adjustment, and stratified analysis, they did not consider disease severity or other issues related to clinical outcomes, which is an important limitation. Nevertheless, recent data from systematic reviews and meta-analyses support the continued use of digoxin as a heart rate control treatment for older patients. ${ }^{35)}$

In clinical practice in Thailand, the decision to prescribe digoxin depends on the patient's characteristics as well as the physician's experience. Despite the lack of statistical significance, patients treated with oral digoxin were likely to have a longer survival time compared to those who did receive digoxin. The current AF guidelines recommend digoxin as a second-line therapeutic agent for heart rate control., ${ }^{9,11)}$ Our findings extend the knowledge that oral digoxin treatment for older patients with both $\mathrm{HF}$ and $\mathrm{AF}$ is safe when used at a proper dose $(\leq 0.125 \mathrm{mg} /$ day $)$.

This study has some limitations. First, this was a single-center study of a limited number of patients. Therefore, these findings cannot be generalized to other hospitals as the patient characteristics and prescribing patterns might vary. Although we tried to include all eligible patients, our sample size was adequate only for the primary objective and not the secondary objective. Second, we lacked data on HF severity (e.g., NYHA or BNP) and clinical status (e.g., heart rate, systolic blood pressure, diastolic blood pressure), which are potential factors associated with both digoxin prescription and clinical outcomes. Third, unlike RCTs, this observational study could not entirely explain the causal relationship because digoxin treatment was not randomly assigned to the patients. Although we adjusted for the difference in baseline characteristics between the two groups, the associations may also be affected by other residual confounding factors. Finally, we could not assess the association of clinical outcomes with the serum level of digoxin due to a lack of data on digoxin levels. Nevertheless, no patient was diagnosed with T46.0 (defined as digoxin toxicity) during the follow-up period.

In conclusion, the use of digoxin in clinical practice was not uncommon among older Thai patients with $\mathrm{HF}$ and AF. The factors negatively associated with digoxin use included higher serum creatinine level and IHD, whereas the positively associated factors included the use of diuretics or mineralocorticoid receptor antagonists. We observed no significant association between digoxin use and the risk of all-cause mortality and rehospitalization due to $\mathrm{HF}$.

\section{ACKNOWLEDGEMENTS}

The authors wish to thank all hospital staff for their support.

\section{CONFLICT OF INTEREST}

The researchers claim no conflicts of interest.

\section{FUNDING}

This study was supported by the School of Pharmaceutical Sciences, University of Phayao (No. PHAR 002/2020) and the University of Phayao (No. FF64-UoE018), Phayao, Thailand.

\section{AUTHOR CONTRIBUTION}

Conceptualization, NS; Data curation, YW, DP, SP, CS; Formal analysis, NS, YW; Investigation, DP, SP, CS; Methodology, NS, YW; Visualization, NS, YW; Project administration, NS; Supervi- 
sion, NS; Writing-original draft, NS; Writing-review \& editing, NS, YW.

\section{REFERENCES}

1. Kazemian P, Oudit G, Jugdutt BI. Atrial fibrillation and heart failure in the elderly. Heart Fail Rev 2012;17:597-613.

2. Santhanakrishnan R, Wang N, Larson MG, Magnani JW, McManus DD, Lubitz SA, et al. Atrial fibrillation begets heart failure and vice versa: temporal associations and differences in preserved versus reduced ejection fraction. Circulation 2016;133: 484-92.

3. Karnik AA, Gopal DM, Ko D, Benjamin EJ, Helm RH. Epidemiology of atrial fibrillation and heart failure: a growing and important problem. Cardiol Clin 2019;37:119-29.

4. Laothavorn P, Hengrussamee K, Kanjanavanit R, Moleerergpoom W, Laorakpongse D, Pachirat O, et al. Thai acute decompensated heart failure registry (Thai ADHERE). CVD Prev Control 2010;5:89-95.

5. Phrommintikul A, Detnuntarat P, Prasertwitayakij N, Wongcharoen W. Prevalence of atrial fibrillation in Thai elderly. J Geriatr Cardiol 2016; 13:270-3.

6. McDonagh TA, Metra M, Adamo M, Gardner RS, Baumbach A, Bohm M, et al. 2021 ESC Guidelines for the diagnosis and treatment of acute and chronic heart failure. Eur Heart J 2021;42: 3599-726.

7. Buakhamsri A, Chirakarnjanakorn S, Sanguanwong S, Porapakkham P, Kanjanavanich R. Heart Failure Council of Thailand (HFCT) 2019 heart failure guideline: pharmacologic treatment of chronic heart failure-part I. J Med Assoc Thai 2019;102:2404.

8. Yingchoncharoen T, Kanjanavanich R. Heart Failure Council of Thailand (HFCT) 2019 heart failure guideline: pharmacologic treatment of chronic heart failure-part II. J Med Assoc Thai 2019; 102:368-72.

9. Chantrarat T, Kunjara Na Ayudhya R, Phrominthikul A, Ariyachaipanich A, Krittayaphong R. Heart Failure Council of Thailand (HFCT) 2019 heart failure guideline: atrial fibrillation in heart failure guidelines. J Med Assoc Thai 2019;102:513-7.

10. Ponikowski P, Voors AA, Anker SD, Bueno H, Cleland JG, Coats AJ, et al. 2016 ESC Guidelines for the diagnosis and treatment of acute and chronic heart failure: the Task Force for the diagnosis and treatment of acute and chronic heart failure of the European Society of Cardiology (ESC)Developed with the special contribution of the Heart Failure Association (HFA) of the ESC. Eur Heart J 2016;37:2129-200.

11. Hindricks G, Potpara T, Dagres N, Arbelo E, Bax JJ, Blom-
strom-Lundqvist C, et al. 2020 ESC Guidelines for the diagnosis and management of atrial fibrillation developed in collaboration with the European Association for Cardio-Thoracic Surgery (EACTS): the Task Force for the diagnosis and management of atrial fibrillation of the European Society of Cardiology (ESC) Developed with the special contribution of the European Heart Rhythm Association (EHRA) of the ESC. Eur Heart J 2021;42: 373-498.

12. O’Mahony D, O’Sullivan D, Byrne S, O'Connor MN, Ryan C, Gallagher P. STOPP/START criteria for potentially inappropriate prescribing in older people: version 2. Age Ageing 2015; 44:213-8.

13. Singh S, Moore H, Karasik PE, Lam PH, Wopperer S, Arundel C, et al. Digoxin initiation and outcomes in patients with heart failure (HFrEF and HFpEF) and atrial fibrillation. Am J Med 2020;133:1460-70.

14. Gao Y, Chang S, Du X, Dong J, Xu X, Zhou Y, et al. Association between digoxin use and adverse outcomes among patients in the Chinese atrial fibrillation registry. Am J Cardiovasc Drugs 2019;19:579-87.

15. Digitalis Investigation Group. The effect of digoxin on mortality and morbidity in patients with heart failure. N Engl J Med 1997;336:525-33.

16. Rich MW, McSherry F, Williford WO; Yusuf S; Digitalis Investigation Group. Effect of age on mortality, hospitalizations and response to digoxin in patients with heart failure: the DIG study. J Am Coll Cardiol 2001;38:806-13.

17. Whitbeck MG, Charnigo RJ, Khairy P, Ziada K, Bailey AL, Zegarra MM, et al. Increased mortality among patients taking digoxin: analysis from the AFFIRM study. Eur Heart J 2013;34: 1481-8.

18. Lopes RD, Rordorf R, De Ferrari GM, Leonardi S, Thomas L, Wojdyla DM, et al. Digoxin and mortality in patients with atrial fibrillation. J Am Coll Cardiol 2018;71:1063-74.

19. Elayi CS, Shohoudi A, Moodie E, Etaee F, Guglin M, Roy D, et al. Digoxin, mortality, and cardiac hospitalizations in patients with atrial fibrillation and heart failure with reduced ejection fraction and atrial fibrillation: an AF-CHF analysis. Int J Cardiol 2020;313:48-54.

20. Turakhia MP, Santangeli P, Winkelmayer WC, Xu X, Ullal AJ, Than CT, et al. Increased mortality associated with digoxin in contemporary patients with atrial fibrillation: findings from the TREAT-AF study. J Am Coll Cardiol 2014;64:660-8.

21. Gheorghiade M, Fonarow GC, van Veldhuisen DJ, Cleland JG, Butler J, Epstein AE, et al. Lack of evidence of increased mortality among patients with atrial fibrillation taking digoxin: findings from post hoc propensity-matched analysis of the AFFIRM trial. 
Eur Heart J 2013;34:1489-97.

22. Allen LA, Fonarow GC, Simon DN, Thomas LE, Marzec LN, Pokorney SD, et al. Digoxin use and subsequent outcomes among patients in a contemporary atrial fibrillation cohort. J Am Coll Cardiol 2015;65:2691-8.

23. Al-Zakwani I, Panduranga P, Zubaid M, Sulaiman K, Rashed WA, Alsheikh-Ali AA, et al. Impact of digoxin on mortality in patients with atrial fibrillation stratified by heart failure: findings from gulf survey of atrial fibrillation events in the middle east. J Cardiovasc Pharmacol Ther 2016;21:273-9.

24. Lam PH, Bhyan P, Arundel C, Dooley DJ, SheriffHM, Mohammed SF, et al. Digoxin use and lower risk of 30-day all-cause readmission in older patients with heart failure and reduced ejection fraction receiving $\beta$-blockers. Clin Cardiol 2018;41:406-12.

25. Qamer SZ, Malik A, Bayoumi E, Lam PH, Singh S, Packer M, et al. Digoxin use and outcomes in patients with heart failure with reduced ejection fraction. Am J Med 2019;132:1311-9.

26. Chao TF, Liu CJ, Chen SJ, Wang KL, Lin YJ, Chang SL, et al. Does digoxin increase the risk of ischemic stroke and mortality in atrial fibrillation? A nationwide population-based cohort study. Can J Cardiol 2014;30:1190-5.

27. Zhou J, Cao J, Jin X, Zhou J, Chen Z, Xu D, et al. Digoxin is associated with worse outcomes in patients with heart failure with reduced ejection fraction. ESC Heart Fail 2020;7:138-46.
28. Knodel J, Chayovan N. Older persons in Thailand: a demographic, social and economic profile. Ageing Int 2008;33:3-14.

29. Charlson ME, Pompei P, Ales KL, MacKenzie CR. A new method of classifying prognostic comorbidity in longitudinal studies: development and validation. J Chronic Dis 1987;40:373-83.

30. Quan H, Sundararajan V, Halfon P, Fong A, Burnand B, Luthi JC, et al. Coding algorithms for defining comorbidities in ICD-9CM and ICD-10 administrative data. Med Care 2005;43:11309.

31. Jenghua K, Jedsadayanmata A. Rate and predictors of early readmission among Thai patients with heart failure. J Med Assoc Thai 2011;94:782-8.

32. Senaviratna NAMR, A. Cooray TMJ. Diagnosing multicollinearity of logistic regression model. Asian J Probab Stat 2019;5:1-9.

33. Ehle M, Patel C, Giugliano RP. Digoxin: clinical highlights: a review of digoxin and its use in contemporary medicine. Crit Pathw Cardiol 2011;10:93-8.

34. Pandya L, Brown DL. The association of digoxin with mortality in ischemic heart failure: a secondary analysis of the STICH trial. Eur HeartJ 2020;41(Suppl_2):ehaa946-0882.

35. Ziff OJ, Lane DA, Samra M, Griffith M, Kirchhof P, Lip GY, et al. Safety and efficacy of digoxin: systematic review and meta-analysis of observational and controlled trial data. BMJ 2015;351: h4451. 